\title{
OTUB1 knockdown promotes apoptosis in melanoma cells by upregulating TRAIL expression
}

\author{
Bok-Soon Lee ${ }^{1, \#}$, Sung Un Kang ${ }^{1, \#}$, Mei Huang ${ }^{1,2}$, Yeon Soo Kim ${ }^{3}$, Young-Sun Lee ${ }^{4}$, Jae-Yong Park ${ }^{4}$ E Chul-Ho Kim ${ }^{1,5, *}$ \\ ${ }^{1}$ Department of Otolaryngology, School of Medicine, Ajou University, ${ }^{2}$ Department of Biomedical Sciences, Ajou University Graduate \\ School of Medicine, Suwon 16499, ${ }^{3}$ Department of Otorhinolaryngology, College of Medicine, Konyang University Hospital, Konyang \\ University Myunggok Medical Research Institute, Daejeon 35365, ${ }^{4}$ School of Biosystem and Biomedical Science, College of Health Science, \\ Korea University, Seoul 02841, ${ }^{5}$ Department of Molecular Science and Technology, Ajou University, Suwon 16499, Korea
}

\begin{abstract}
Melanoma, the most serious type of skin cancer, exhibits a high risk of metastasis. Although chemotherapeutic treatment for metastatic melanoma improves disease outcome and patient survival, some patients exhibit resistance or toxicity to the drug treatment regime. OTUB1 is a deubiquitinating enzyme overexpressed in several cancers. In this study, we investigated the effects of inhibiting OTUB1 expression on melanoma-cell proliferation and viability and identified the underlying molecular mechanism of action of OTUB1. We did endogenous OTUB1 knockdown in melanoma cells using short interfering RNA, and assessed the resulting phenotypes via MTT assays, Westem blotting, and cell-cycle analysis. We identified differentially expressed genes between OTUB1-knockdown cells and control cells using RNA sequencing and confirmed them via Westem blotting and reverse transcription polymerase chain reaction. Furthermore, we investigated the involvement of apoptotic and cell survival signaling pathways upon OTUB1 depletion. OTUB1 depletion in melanoma cells decreased cell viability and caused simultaneous accumulation of cells in the sub-G1 phase, indicating an increase in the apoptotic-cell population. RNA sequencing of OTUB1-knockdown cells revealed an increase in the levels of the apoptosis-inducing protein TRAIL. Additionally, OTUB1-knockdown cells exhibited increased sensitivity to PLX4032, a BRAF inhibitor, implying that OTUB1 and BRAF act collectively in regulating apoptosis. Taken together, our findings show that OTUB1 induces apoptosis of melanoma cells in vitro, likely by upregulating TRAIL, and suggest that approaches targeting OTUB1 can be developed to provide novel therapeutic strategies for treating melanoma. [BMB Reports 2021; 54(12): 608-613]
\end{abstract}

*Corresponding author. Tel: +82-31-219-5269; Fax: +82-31-219-5264; E-mail: ostium@ajou.ac.kr

${ }^{\#}$ These authors contributed equally to this work.

https://doi.org/10.5483/BMBRep.2021.54.12.033

Received 3 March 2021, Revised 23 March 2021, Accepted 2 August 2021

Keywords: Cell death, Melanoma, OTUB1, PLX4032, TRAIL

\section{INTRODUCTION}

Melanoma, an aggressive cancer of the skin or mucosa, is the fifth most-common cancer in men and the sixth in women in the United States (1). Although the incidence of most solid tumors has decreased or at least stabilized in the last few decades, the incidence of skin cancer has continued to increase worldwide, particularly affecting the light-skinned Caucasian populations exposed to excessive UV radiation (2). Melanoma is the most lethal skin cancer and accounts for $75 \%$ of deaths caused by skin cancers (3). Therefore, chemotherapy, immunotherapy, and gene therapy are being intensely investigated as cures for metastatic malignant melanoma; however, long-term cures are rare, primarily because of refractory brain metastases (4).

Ovarian tumor domain containing ubiquitin aldehyde-binding protein 1 (OTUB1; also called otubain 1) belongs to the ovarian tumor domain protease subfamily of deubiquitinating enzymes (DUBs) (5). OTUB1 was first identified as an inhibitor of doublestrand break-induced chromatin ubiquitination (6). In addition to its role of direct removal of ubiquitin from substrates, OTUB1 can inhibit polyubiquitination of target proteins by blocking the E2/E3 ligase-dependent ubiquitin transfer. Juang et al. reported that OTUB1 is a lysine 48 linkage-specific deubiquitinating enzyme and elucidated the structural mechanism by which OTUB1 binds E2 ligases to inhibit ubiquitin transfer (7).

Many studies have reported that OTUB1 is related to the progression and aggressiveness of lung cancer (8), colorectal cancer (9), prostate cancer (10), breast cancer (11), gastric cancer (12), hepatocellular carcinoma (HCC) (13), esophageal squamous cell carcinoma (14), and glioma (15). However, the exact mechanisms underlying OTUB1-mediated regulation of melanoma progression have not been reported.

Tumor necrosis factor (TNF)-related apoptosis-inducing ligand or Apo 2 ligand (TRAIL/Apo2L) is a member of the TNF family that usually induces apoptosis in several kinds of cancer by engaging with its receptors DR4 and DR5 on the cell membrane (16). Once TRAIL binds to its receptor, receptor oligomerization recruits downstream molecules such as FADD, subsequently leading to the formation of procaspase 8 and activating the death-inducing signaling complex (17). Although melanoma cells

ISSN: 1976-670X (electronic edition)

Copyright (C) 2021 by the The Korean Society for Biochemistry and Molecular Biology

(c) This is an open-access article distributed under the terms of the Creative Commons Attribution Non-Commercial License (http://creativecommons.org/licenses/by-nc/4.0) which permits unrestricted non-commercial use, distribution, and reproduction in any medium, provided the original work is properly cited. 
constitutively express DR5, they display various degrees of sensitivity to TRAIL (18). Most melanoma tissues express both receptors, suggesting that initial TRAIL sensitivity is characteristic for clinical melanoma.

In this study, we show that OTUB1 knockdown in human melanoma cells promotes cell death by upregulating TRAIL expression. Our findings provide insight into the mechanism of OTUB1mediated cell death in melanoma and indicate OTUB1 as a potential therapeutic target for inhibiting melanoma progression.

\section{RESULTS}

OTUB1 is highly expressed in melanoma cancer-cell lines First, we identified the expression of OTUB1 in several cancercell lines (lung cancer, gastric cancer, colon cancer, HCC, and melanoma) via Western blotting. Of the cell lines we used, OTUB1 was highly expressed in melanoma-cell lines (Fig. 1A). Second, we observed the effect of OTUB1 expression on survival in melanoma patients using The Cancer Genome Atlas (TCGA) data to obtain Kaplan-Meier plots. Interestingly, we discovered that melanoma patients with high OTUB1 expression had a worse prognosis than did those with low OTUB1 expression (Fig. 1B).

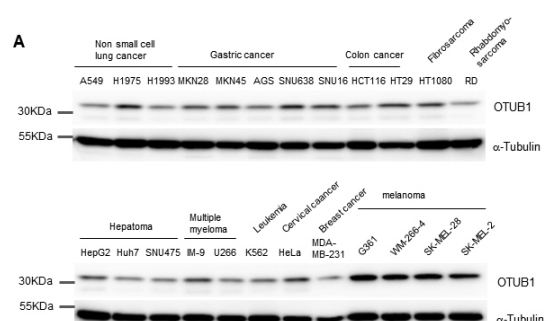

B

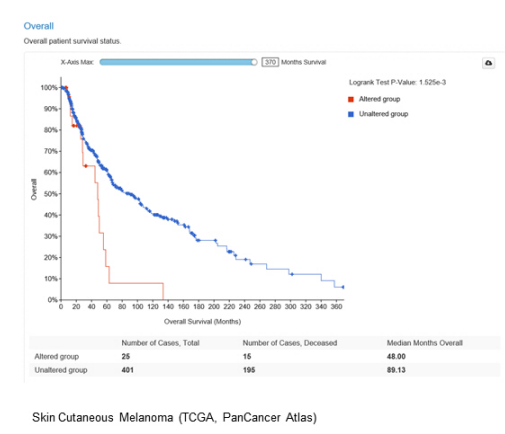

Fig. 1. OTUB1 is overexpressed in melanoma-cell lines. (A) We harvested actively proliferating cells in several cancer-cell lines and did Western blotting for OTUB1, using $\alpha$-Tubulin as a loading control. (B) KaplanMeier survival curves of patients showing the effect of low or high OTUB1 expression on overall survival rates.

\section{OTUB1 knockdown induces apoptosis in melanoma cells and inhibits cell-cycle progression}

To verify the role of OTUB1 in melanoma cell lines and its relationship with apoptosis and cell cycles during cancer progression, we depleted endogenous OTUB1 using short interfering RNA (siRNA) and examined the resulting phenotypes by MTT assay, Annexin V-PI staining, and cell-cycle analysis. Microscopic analysis showed an increase in death of G361 and WM-266-4 cells 48 and $72 \mathrm{~h}$ after OTUB1 knockdown over that in the control cells (Fig. 2A). The MTT assay results also showed a reduction in cell viability after OTUB1 knockdown (Fig. 2B). Using Annexin V-PI staining, we then assessed whether the reduction in cell viability resulted from an increase in apoptosis. We observed much more apoptosis in OTUB1-knockdown cells than in the control cells (Fig. 2C). The fluorescence-activated cell-sorting experiment confirmed our finding of the presence of apoptotic cells and enabled us to examine the distribution of cells in different cell-cycle phases. OTUB1-depleted WM-266-4 cells accumulated in the G1 phase; this finding strongly supports the evidence that OTUB1 depletion in melanoma cells triggers apoptosis (Fig. 2D).

To further support our hypothesis of apoptosis by OTUB1 knockdown, we subjected WM-266-4 cells to Z-VAD treatment with siRNA for OTUB1. The cells were stained with Annexin V-FITC and propidium iodide, followed by analysis using FACS (Fig. 2E, F) and Western blotting (Fig. 2G). The results indicate that OTUB1 knockdown-induced apoptotic cell death was caspase-dependent.

TRAIL expression is upregulated in OTUB1-knockdown cells Next, we used the PathScan Stress and Apoptosis Signaling Antibody Array to find out the relationship between OTUB1 knockdown-activated cell signaling pathways and apoptosis. It is well known that pp38 is activated in the TRAIL pathway and that apoptosis markers, such as cleaved PARP or cleaved caspase 3, increase during apoptosis $(19,20)$. The results of the apoptosis assay revealed that phosphorylation of apoptotic proteins, such as Bad, p38 mitogen-activated protein kinase (MAPK), caspase 3, and Chk2, was increased more in OTUB1-knockdown cells than in the control cells (Fig. 3A). We also assessed the survival-related cell signaling using the RTK array and found that the basal phosphorylation levels of signaling proteins, such as c-MET, ROR2, and EphA10, were more reduced in OTUB1-knockdown cells than in the control cells (Fig. 3B). Furthermore, RNA sequencing showed significantly more TRAIL mRNA in OTUB1-knockdown cells than in the control cells (Fig. 3C) in accordance with the increase in apoptosis previously observed. Taken together, we conclude that OTUB1 knockdown reduces cell survival-related signaling and simultaneously activates apoptosis-related signaling pathways, thereby triggering apoptosis, potentially by upregulating the apoptosis-inducing protein TRAIL. 


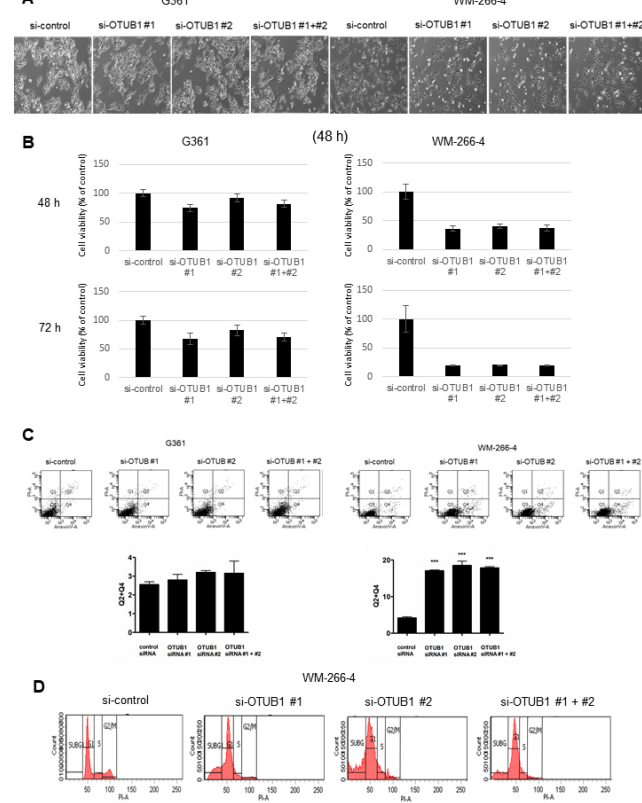

E
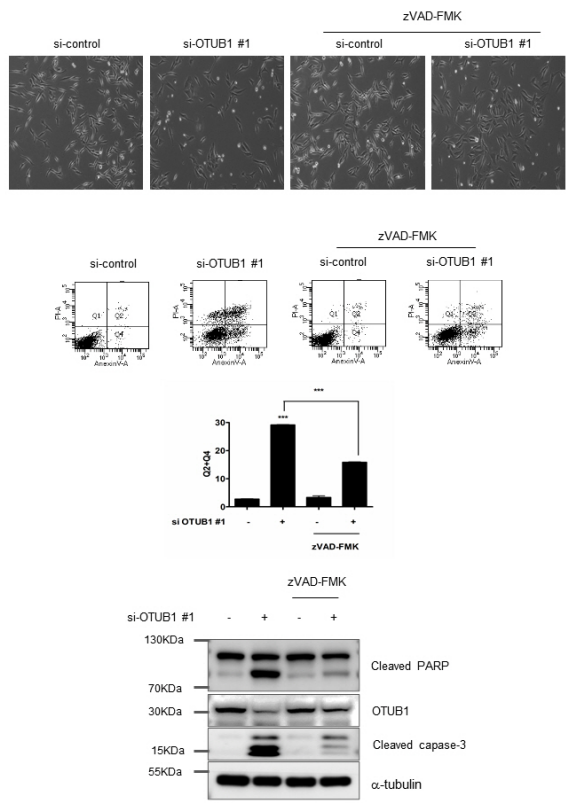

Fig. 2. Knockdown of OTUB1 by siRNA inhibits cell proliferation and induces apoptosis. (A) We examined cell death after OTUB1 knockdown using a light microscope. (B) We quantified cell viability after OTUB1 knockdown using the MTT assay. (C) Cells were stained with Annexin V-FITC/PI and analyzed using flow cytometry. (D) Distribution of cells across the different cell-cycle phases. (E) Cells were pretreated with Z-VAD, followed by treatment with siRNA for OTUB1, and then examined using a light microscope. (F) Cells were stained with Annexin V-FITC/PI and analyzed using flow cytometry. (G) We did Western blotting antibodies against OTUB1, cleaved caspase 3, and cleaved PARP.

A
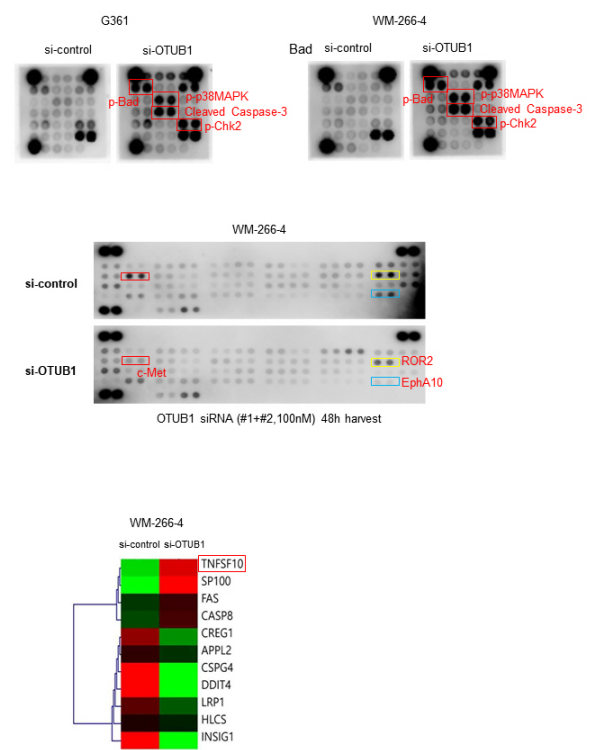

Fig. 3. TRAIL is upregulated in OTUB1-knockdown cells. (A) G361 or WM-266-4 melanoma-cell lines were transfected with OTUB1 siRNA for $24 \mathrm{~h}$. PathScan Stress and Apoptosis Signaling Antibody Array. (B) The RTK array. (C) RNA sequencing of WM-266-4 cells treated (or not) with OTUB1 siRNA. A heat map representing differential gene expression between control and OTUB1-knockdown cells is shown.

\section{Upregulation of TRAIL in OTUB1-knockdown cells increases apoptosis}

We further confirmed the RNA-sequencing results by evaluating the expression of TRAIL using reverse transcription polymerase chain reaction (RT-PCR) in G361, WM-266-4, SK-MEL-28, and SK-MEL-5 cell lines. Three cell lines exhibited higher expression of TRAIL in OTUB1-knockdown cells than in control cells (Fig. 4A). A previous study reported that increasing the expression of TRAIL receptors affected the sensitivity of TRAIL-induced apoptosis (21). However, it is unclear whether OTUB1 knockdown also alters the expression of DR4 or DR5. There was no change in the mRNA levels of DR4 or DR5 in OTUB1-knockdown cells according to our PCR analysis on melanoma cells with OTUB1 knockdown (Fig. 4A). Moreover, according to our Western blotting, the OTUB1-depleted G361 and WM-266-4 cells showed the presence of apoptosis effectors, such as cleaved caspase 3 and cleaved poly (ADP-ribose) polymerase (PARP) (Fig. 4B). To further investigate the presence of TRAIL, which possibly increases apoptosis in the absence of OTUB1, we exogenously added TRAIL to OTUB1-knockdown cells. As expected, TRAIL induced apoptosis in OTUB1-knockdown cells more than in control G361 and WM-266-4 cell lines (Fig. 4C).

It is known that PLX4032, or vemurafenib, an FDA-approved drug for the treatment of melanoma, inhibits mutant BRAF $\left(\mathrm{BRAF}^{\mathrm{V} 600 \mathrm{E}}\right)$. Here, we suggested that PLX4032 treatment could increase apoptosis in cells lacking OTUB1. Therefore, we treated 
A

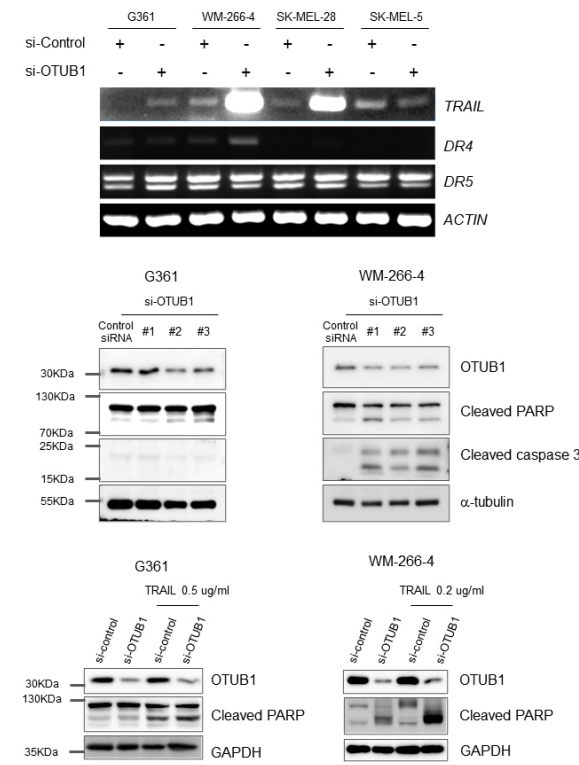

D
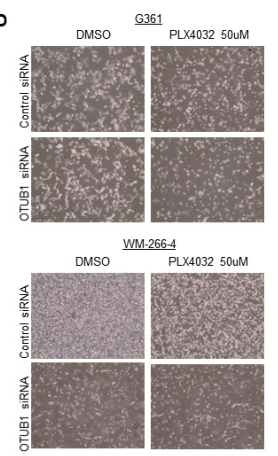

E
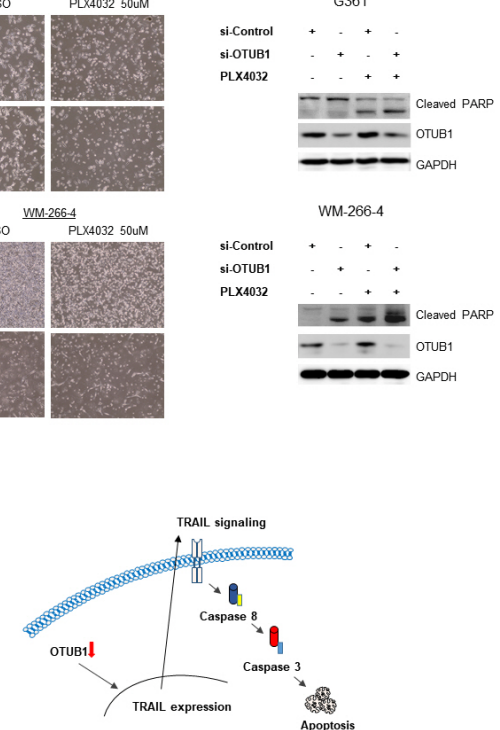

Fig. 4. OTUB1 knockdown upregulates the expression of TRAIL in melanoma-cell lines. (A) The OTUB1-knockdown melanoma-cell lines were harvested and analyzed using RT-PCR. (B) G361 and WM-266-4 cells were transfected with three different siRNAs (\#1, \#2, \#3) targeting OTUB1. We did Western blotting. (C) G361 and WM-266-4 cells were transfected with OTUB1 siRNA and treated with TRAIL $(0.5$ or $0.2 \mu \mathrm{g} / \mathrm{ml})$. We did Western blotting. (D) G361 and WM-266-4 cells were transfected with OTUB1 siRNA and then treated with PLX4032 (50 uM) for $24 \mathrm{~h}$. We examined cell death using a light microscope. (E) We did Western blotting using antibodies against OTUB1 and used cleaved PARP; GAPDH as a loading control. (F) A proposed model for OTUB1 knockdown-induced cell death through TRAIL expression.

OTUB1-depleted G361 and WM-266-4 with PLX4032 for $24 \mathrm{~h}$ and measured the cell proliferation. According to microscopic analysis, PLX4032-induced cell death was increased in OTUB1knockdown cells more than in cells treated with control siRNA (Fig. 4D), and the synergism between PLX4032 treatment and OTUB1 depletion on the control of apoptosis was also observed by an increase in cleaved PARP levels in OTUB1-knockdown cells treated with PLX4032 (Fig. 4E). Collectively, our results indicate that the strategies targeting the regulation of OTUB1 expression in melanoma may be valuable in achieving successful therapeutic intervention.

\section{DISCUSSION}

In this study, we showed that OTUB1 knockdown in melanoma cells induces apoptotic cell death by upregulating TRAIL (Fig. 4F). To our knowledge, this is the first study showing that strategically targeting the expression of OTUB1 in melanoma may possess therapeutic potential. Several studies have reported that OTUB1 affects overall cancer progression by regulating the stability of target oncogenes because of its role as a DUB (8, $11,22)$. It is highly expressed in many cancers and is known to inhibit the degradation of molecules that affect cell proliferation, such as Ras (8), FOXM1 (23), c-Maf $(24,25)$, and c-IAP1 $(26,27)$. However, we could not identify the exact molecular target of OTUB1 and therefore could not elucidate the mecha- nism of OTUB1 in regulating melanoma-cell apoptosis. However, as a direct evidence of the occurrence of apoptosis, we showed that expression of TRAIL increases at the transcription level. OTUB1 has been proposed to promote the progression of myeloma (24), colorectal (9), prostate cancer (10), ovarian cancer (28), gastric cancer (12), HCC (12), and glioma (15) by facilitating tumor invasiveness. Furthermore, OTUB1 acts as a potential distant metastasis marker and prognostic factor in colorectal cancer (9). In addition to melanoma, OTUB1 is highly expressed in HCC (13); however, the inhibition of OTUB1 expression reduced the progression of HCC. Taken together, OTUB1 is highly expressed in various cancers and is a potential target to prevent cancer progression and metastasis.

According to a recent study, inducible deletion of OTUB1 in adult mice greatly decreased tumor growth; this effect was associated with infiltration of various immune cells, including $\mathrm{CD}^{+}{ }^{+} \mathrm{T}$ cells, NK cells, CD4 ${ }^{+} \mathrm{T}$ cells, and $\mathrm{cDC} 1$ cells (29). When the expression of OTUB1 is inhibited, the activation of immune cells to kill cancer cells is interpreted as a good point. Although we have not confirmed it, this may play a large role in reducing the tumor size of melanomas. Interestingly, our results also show that OTUB1 downregulation significantly induced apoptosis in melanoma cells. However, from a clinical point of view, further investigation via in vitro and in vivo studies is required to establish OTUB1 as a potential target for melanoma treatment. 
Our data show that all melanoma-cell lines used in this study (G361, WM-266-4, SK-MEL-28, and SK-MEL-5) had higher expression of OTUB1 than did any other cancer-cell lines (Fig. 1A). As seen from TCGA data, the high expression of OTUB1 in melanoma correlated with poor patient survival (Fig. 1B), pointing towards the potential use of OTUB1 in melanoma as a therapeutic target.

The data of PathScan Stress and Apoptosis Signaling Antibody Array between G361 and WM-266-4 cells were similar, because the array is sensitive; however, the results of Western blotting for both cells were markedly different (Fig. 3A). In G361 cells, OTUB1 knockdown showed little TRAIL expression, whereas a much larger increase in TRAIL expression was observed when OTUB1 was knocked down in WM-266-4 cells (Fig. 4A). Therefore, we believe that the difference in apoptosis can be attributed to the difference in TRAIL expression that leads to apoptosis upon OTUB1 knockdown.

Most melanomas require activation of the MAPK pathway, which is most often triggered by an oncogenic mutant of BRAF $\left(\mathrm{BRAF}^{\mathrm{V} 600 \mathrm{E}}\right)(30,31)$. Consistently, mutant BRAF inhibitors such as PLX4032 (vemurafenib) are used to treat many melanoma patients (31). The overall response rate of patients with $\mathrm{BRAF}^{\mathrm{V} 600 \mathrm{E}}$ positive melanomas to vemurafenib is approximately $50 \%$, with responsive patients displaying substantial tumor regression and prolonged survival (32).

In this study, OTUB1 and BRAF exercised a synergism on the regulation of apoptosis. Furthermore, OTUB1 overexpression reversed the apoptosis induced by the inhibition of BRAF, pointing towards a potential mechanism of cancer recurrence and suggesting that the expression of OTUB1 is essential for melanoma-cell survival. Therefore, this study ultimately sheds light on the potential mechanism behind how OTUB1 expression promotes cancer survival and proposes that inhibition of OTUB1 expression can supplement existing treatment approaches for melanoma.

\section{MATERIALS AND METHODS}

Futher details on the analysis methods are described in Supplementary Materials and Methods.

\section{Cell culture and reagents}

Human melanoma cell lines (G361, WM-266-4, SK-MEL-2, SKMEL-5, SK-MEL-28) were purchased from Korean Cell Line Bank (Seoul, South Korea). G361, SK-MEL-2 and SK-MEL-5 were cultured in RPMI and WM-266-4 and SK-MEL-28 were cultured in minimum essential medium (MEM) (Welgene Inc., South Korea). The cell lines were maintained at $37^{\circ} \mathrm{C}$ and $5 \%$ $\mathrm{CO}_{2}$ in media supplemented with $10 \%$ fetal bovine serum (Welgene Inc, South Korea) and 1\% of antibiotics (Gibco/Invitrogen, Carlsbad, CA). The PathScan Stress and Apoptosis Signaling Antibody Array Kit (\#12856) was purchased from Cell Signaling Technology (Danvers, MA, USA). Human Phospho-Receptor Tyrosine Kinase (RTK) Array Kit (\#ARY001B) were purchased from R\&D Systems (Minneapolis, MN, USA).

\section{ACKNOWLEDGEMENTS}

This research was supported by a grant of the Korea Health Technology R\&D Project through the Korea Health Industry Development Institute (KHIDI), funded by the Ministry of Health \& Welfare, Republic of Korea (Grant No: HR21C1003), and was supported by the National Research Foundation of Korea (Grant No: 2017R1D1A1B03028527 and 2018R1A2B3009008).

\section{CONFLICTS OF INTEREST}

The authors have no conflicting interests.

\section{REFERENCES}

1. Rigel DS, Friedman RJ and Kopf AW (1996) The incidence of malignant melanoma in the United States: issues as we approach the 21st century. J Am Acad Dermatol 34, 839-847

2. D'Orazio J, Jarrett S, Amaro-Ortiz A et al (2013) UV radiation and the skin. Int J Mol Sci 14, 12222-12248

3. Laikova KV, Oberemok VV, Krasnodubets AM et al (2019) Advances in the understanding of skin cancer: ultraviolet radiation, mutations, and antisense oligonucleotides as anticancer drugs. Molecules 24, 1516

4. Domingues B, Lopes JM, Soares P et al (2018) Melanoma treatment in review. Immunotargets Ther 7, 35-49

5. Shan TL, Tang ZL, Guo DZ et al (2009) Partial molecular cloning, characterization, and analysis of the subcellular localization and expression patterns of the porcine OTUB1 gene. Mol Biol Rep 36, 1573-1577

6. Nakada S, Tai I, Panier S et al (2010) Non-canonical inhibition of DNA damage-dependent ubiquitination by OTUB1. Nature 466, 941-946

7. Juang YC, Landry MC, Sanches M et al (2012) OTUB1 co-opts Lys48-linked ubiquitin recognition to suppress E2 enzyme function. Mol Cell 45, 384-397

8. Baietti MF, Simicek M, Abbasi Asbagh L et al (2016) OTUB1 triggers lung cancer development by inhibiting RAS monoubiquitination. EMBO Mol Med 8, 288-303

9. Zhou Y, Wu J, Fu X et al (2014) OTUB1 promotes metastasis and serves as a marker of poor prognosis in colorectal cancer. Mol Cancer 13, 258

10. Iglesias-Gato D, Chuan YC, Jiang N et al (2015) OTUB1 de-ubiquitinating enzyme promotes prostate cancer cell invasion in vitro and tumorigenesis in vivo. Mol Cancer 14,8

11. Karunarathna $U$, Kongsema M, Zona $S$ et al (2016) OTUB1 inhibits the ubiquitination and degradation of FOXM1 in breast cancer and epirubicin resistance. Oncogene 35, 1433-1444

12. Weng W, Zhang Q, Xu M et al (2016) OTUB1 promotes tumor invasion and predicts a poor prognosis in gastric adenocarcinoma. Am J Transl Res 8, 2234-2244

13. Ni Q, Chen J, Li X et al (2017) Expression of OTUB1 in hepatocellular carcinoma and its effects on HCC cell migration and invasion. Acta Biochim Biophys Sin (Shanghai) 
$49,680-688$

14. Zhou H, Liu Y, Zhu R et al (2018) OTUB1 promotes esophageal squamous cell carcinoma metastasis through modulating Snail stability. Oncogene 37, 3356-3368

15. Xu L, Li J, Bao Z et al (2017) Silencing of OTUB1 inhibits migration of human glioma cells in vitro. Neuropathology 37, 217-226

16. Schneider $P$, Thome $M$, Burns $K$ et al (1997) TRAIL receptors 1 (DR4) and 2 (DR5) signal FADD-dependent apoptosis and activate NF-kappaB. Immunity 7, 831-836

17. Wang S and El-Deiry WS (2003) TRAIL and apoptosis induction by TNF-family death receptors. Oncogene 22, 8628-8633

18. Kurbanov BM, Geilen CC, Fecker LF et al (2005) Efficient TRAIL-R1/DR4-mediated apoptosis in melanoma cells by tumor necrosis factor-related apoptosis-inducing ligand (TRAIL). J Invest Dermatol 125, 1010-1019

19. Ohtsuka T, Buchsbaum D, Oliver P et al (2003) Synergistic induction of tumor cell apoptosis by death receptor antibody and chemotherapy agent through JNK/p38 and mitochondrial death pathway. Oncogene 22, 2034-2044

20. Kim EY, Ryu JH and Kim AK (2013) CAPE promotes TRAILinduced apoptosis through the upregulation of TRAIL receptors via activation of $\mathrm{p} 38$ and suppression of JNK in SK-Hep1 hepatocellular carcinoma cells. Int J Oncol 43, 1291-1300

21. Zhang XD, Franco A, Myers K et al (1999) Relation of TNF-related apoptosis-inducing ligand (TRAIL) receptor and FLICE-inhibitory protein expression to TRAIL-induced apoptosis of melanoma. Cancer Res 59, 2747-2753

22. Herhaus L, Al-Salihi M, Macartney T et al (2013) OTUB1 enhances TGFbeta signalling by inhibiting the ubiquitylation and degradation of active SMAD2/3. Nat Commun 4,
2519

23. Zhou K, Mai H, Zheng S et al (2020) OTUB1-mediated deubiquitination of FOXM1 up-regulates ECT-2 to promote tumor progression in renal cell carcinoma. Cell Biosci 10, 50

24. Xu Y, Xu M, Tong J et al (2021) Targeting the Otub1/c-Maf axis for the treatment of multiple myeloma. Blood 137, 1478-1490

25. Sun T, Xu Y, Xu Z et al (2021) Inhibition of the Otub1/ c-Maf axis by the herbal acevaltrate induces myeloma cell apoptosis. Cell Commun Signal 19, 24

26. Koschel J, Nishanth G, Just S et al (2021) OTUB1 prevents lethal hepatocyte necroptosis through stabilization of c-IAP1 during murine liver inflammation. Cell Death Differ 28, 2257-2275

27. Goncharov T, Niessen K, de Almagro MC et al (2013) OTUB1 modulates C-IAP1 stability to regulate signalling pathways. EMBO J 32, 1103-1114

28. Wang $Y$, Zhou X, Xu M et al (2016) OTUB1-catalyzed deubiquitination of FOXM1 facilitates tumor progression and predicts a poor prognosis in ovarian cancer. Oncotarget 7, 36681-36697

29. Zhou $X, Y u$ J, Cheng $X$ et al (2019) The deubiquitinase Otub1 controls the activation of CD8(+) T cells and NK cells by regulating IL-15-mediated priming. Nat Immunol 20, 879-889

30. Massa RC and Kirkwood JM (2019) Targeting the MAPK pathway in advanced BRAF wild-type melanoma. Ann Oncol 30, 503-505

31. Cheng Y, Zhang G and Li G (2013) Targeting MAPK pathway in melanoma therapy. Cancer Metastasis Rev 32, 567-584

32. Mandala M and Voit C (2013) Targeting BRAF in melanoma: biological and clinical challenges. Crit Rev Oncol Hematol 87, 239-255 